Unfallchirurg 2012 · 115:574-575

DOI 10.1007/s00113-012-2173-4

c) Springer-Verlag 2012
M. Schädel-Höpfner · J. Windolf

Klinik für Unfall- und Handchirurgie, Universitätsklinikum Düsseldorf

\title{
Besondere traumatische und degenerative Schäden der Hand
}

Das vorliegende dritte handchirurgische Themenheft bildet erneut eine große Bandbreite spannender und aktueller Fragestellungen $\mathrm{ab}$, wobei diesmal besondere traumatische und degenerative Schäden mit den zugehörigen diagnostischen und therapeutischen Konzepten vorgestellt werden. Im Mittelpunkt steht zunächst das Handgelenk. Dieses gilt als das komplizierteste Gelenk des menschlichen Körpers. Die morphologische und funktionelle Integrität des Handgelenks ermöglicht komplexe Bewegungsabläufe und die Positionierung der Hand als Voraussetzung für deren vielfältige Beanspruchung. Die besonderen Funktionen werden durch ein abgestimmtes Zusammenwirken der benachbarten Gelenkabschnitte gewährleistet. $\mathrm{Zu}$ diesen gehören das Radiokarpalgelenk als Handgelenk im engeren Sinne, das Mediokarpalgelenk und das distale Radioulnargelenk. Posttraumatische und degenerative Veränderungen können alle Gelenkabschnitte schädigen, wobei jeweils spezifische Veränderungen mit charakteristischen Störungen existieren. Das aktuelle Themenheft beschäftigt sich deshalb mit Schädigungen in umittelbarer Nachbarschaft des Radiokarpalgelenks und stellt aktuelle Konzepte zu ihrer Behandlung vor.

Perilunäre Luxationen und Luxationsfrakturen sind seltene, aber schwere Handgelenkverletzungen, die sowohl den proximalen als auch den distalen Gelenkabschnitt betreffen und eine vielfältige Morphologie aufweisen können. Während die Herausforderungen einer korrekten Diagnosestellung und adäquaten Erstversorgung zunehmend besser gemeistert werden, ist die operative Therapie immer noch anspruchsvoll und führte häufig zu unbefriedigenden radiologischen Ergebnissen, die jedoch durch günstigere klini- sche Resultate kaschiert werden. Alle Behandlungsempfehlungen zielen auf eine anatomische und stabile Rekonstruktion des Karpus und weisen deshalb eine weitgehende Übereinstimmung auf.

Bei den Läsionen des „triangular fibrocartilaginous complex" (TFCC) ist dagegen die Diagnose schwerer und häufig nur über den operativen Aufwand der Handgelenkarthroskopie zu stellen. Noch problematischer ist es, Therapieempfehlungen für Schäden des TFCC auszusprechen. Die hierzu vorliegenden Publikationen sind uneinheitlich und beinhalten eine Vielzahl von Behandlungskonzepten. Durch eine systematische Literaturauswertung konnten lediglich einige Therapiestrategien identifiziert werden.

Der TFCC dient aber nicht nur der axialen Kraftübertragung vom Unterarm auf den Karpus, sondern ist v. a. auch als Stabilisator des distalen Radioulnargelenks (DRUG) bedeutsam. Eine Arthrose des DRUG entsteht besonders häufig posttraumatisch nach einer distalen Radiusfraktur und führt zu einer schmerzhaften Einschränkung der Unterarmdrehbewegung. Bei hohem Leidensdruck können verschiedene operative Verfahren eingesetzt werden, bei denen das distale Radioulnargelenk entweder reseziert oder versteift wird. Die Resultate sind variabel und beinhalten das Risiko einer radiolulnaren Instabilität. Einen neuen und erfolgversprechenden Ansatz stellen Ulnakopfprothesen dar.

Ein besonders spannendes Thema sind die Schuss- und Explosionsverletzungen der Hand, welche in Mitteleuropa selten beobachtet werden, aber hohe Anforderungen an den behandelnden Chirurgen stellen. Durch Afghanistan-Einsätze verfügten die Autoren des Beitrags über eine eigene kriegschirurgische Expertise.
Gefordert sind hier neben Kenntnissen der Verletzungsentstehung ein konsequentes Management, um Komplikationen zu vermeiden und ein bestmögliches Behandlungsresultat zu erzielen.

Weniger bedrohlich für den Erhalt und die Funktion der gesamten Hand sind die ebenfalls seltenen Bandverletzungen der Fingergrundgelenke. Wegen der Bedeutung einer ungestörten Beweglichkeit dieser Gelenke für die Greiffunktion der Hand bedürfen derartige Bandverletzungen aber sowohl einer gezielten Diagnostik, um nicht übersehen zu werden, als auch einer individuellen Behandlung mit dem Ziel der vollständigen Funktionswiederherstellung.

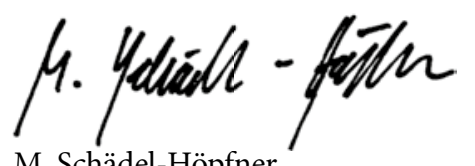

M. Schädel-Höpfner

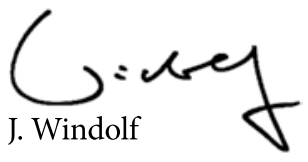

\section{Korrespondenzadressen}

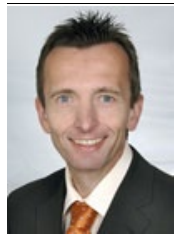

Prof. Dr. M. Schädel-Höpfner Klinik für Unfall- und Handchirurgie, Universitätsklinikum Düsseldorf Moorenstraße 5, 4022 Düsseldorf schaedel@uni-duesseldorf.de

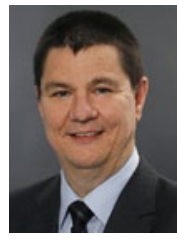

Prof. Dr. J. Windolf Klinik für Unfall- und Handchirurgie Universitätsklinikum Düsseldorf Moorenstraße 5 40225 Düsseldorf windolf@uni-duesseldorf.de 
Hier steht eine Anzeige.

黛 Springer 